\title{
Lateral correlation of SiGe Stranski-Krastanow islands on silicon as probed by high resolution $\mathrm{x}$-ray diffraction
}

\author{
M. Hanke, ${ }^{\text {a) }}$ M. Schmidbauer, and R. Köhler \\ Institut für Physik, Humboldt-Universität zu Berlin, Newtonstraße 15, D-12489 Berlin, Germany
}

(Received 20 January 2004; accepted 23 May 2004)

\begin{abstract}
We describe a procedure to consider the impact of lateral positional correlation of SiGe nanoscale islands onto the diffuse scattering within a high resolution $\mathrm{x}$-ray diffraction experiment. The samples have been grown by means of liquid phase epitaxy which provides monodisperse island ensembles containing up to $10^{9}$ equivalent objects. It is shown that a proper numerical simulation of the x-ray diffuse scattering pattern requires careful consideration of the partial coherence of $x$ rays. An appropriate numerical procedure consists of coherent summation over sample areas with lateral dimensions as given by the coherence properties of the radiation and subsequent incoherent summation over a large enough number of such areas. For the given case an effective lateral coherence length of $\approx 1 \mu \mathrm{m}$ has been used, which is derived taking into account also the detector resolution. The according simulation is in good agreement with the experimentally observed $\mathrm{x}$-ray diffuse intensity pattern. (C) 2004 American Institute of Physics. [DOI: 10.1063/1.1772883]
\end{abstract}

\section{INTRODUCTION}

Low-dimensional structures, as, e.g., quantum dots (QDs) have attracted an overwhelming interest during the last years. ${ }^{1}$ Many dedicated device applications require highly uniform ensembles of such structures. Thus, different routes towards perfectly ordered nanoscale objects have been employed. Self-organization during crystal growth via the so-called Stranski-Krastanow growth mode is one of the most promising approaches. Here, the elastic energy present at heteroepitaxial strained layer growth is decreased by the formation of nanoscale islands allowing for elastic strain relaxation. This process is often accompanied by lateral, ${ }^{2,3}$ vertical, ${ }^{4}$ or even three-dimensional ${ }^{5}$ positional correlation.

Structural investigations of QDs usually start with direct imaging techniques such as scanning electron microscopy, atomic force microscopy and transmission electron microscopy. Those are excellent analytical tools to study shape, size and in case of TEM even chemical composition profiles of usually a few individual objects. ${ }^{6}$ To a limited extent they can also provide information regarding positional correlation. However, while the mentioned imaging techniques excel in a high spatial resolution, they usually probe small ensembles as compared to $\mathrm{X}$-ray scattering techniques as grazing incidence small angle x-ray scattering (GISAXS), grazing incidence diffraction (GID), and high resolution x-ray diffraction (HRXRD). GISAXS does not provide any information about the strain state, however, in a QD it can be applied to exclusively probe shape, size, and positional correlation. ${ }^{7,8}$ Compared to that HRXRD and GID additionally probe small lattice distortions which-in combination with scattering simulations-makes a three-dimensional determination of strain and chemical composition feasible, e.g., Refs. 9-11. Generally the diffusely scattered intensity is af-

\footnotetext{
${ }^{a)}$ Present address: Fachbereich Physik, Martin-Luther-Universität HalleWittenberg, Hoher Weg 8, D-06120 Halle/Saale, Germany; electronic mail: hanke@physik.uni-halle.de
}

fected by both vertical and lateral positional correlation. Consequently the cited $\mathrm{x}$-ray scattering techniques contain information on positional correlation in both spatial directions. ${ }^{12-17}$ Recently a very promising attempt employs anomalous $\mathrm{x}$-ray scattering to enhance the chemical contrast of buried quantum dots in order to investigate ordering properties. ${ }^{18}$ Usually theoretical approaches either restrict themselves to the completely coherent case [see Eq. (1) below] or additionally indicate averaging. In the latter case averaging is then often implicitly part of an analytical correlation function, see, e.g., Ref. 13.

\section{SAMPLE}

Here we will demonstrate the influence of the partial coherence of the applied radiation on x-ray diffuse scattering. For that purpose we have utilized SiGe islands on $\mathrm{Si}(100)$, which serve as a model system with an outstanding degree of conformity in terms of island size, shape, and orientation. The respective samples have been grown by means of liquid phase epitaxy, which operates comparatively close to the thermodynamical equilibrium providing highly monodisperse ensembles, which contain only a single type of dislocation-free nanoscale islands. Almost all of them are made of truncated pyramids with $\{111\}$ side facets and an (001) top facet. Atomic force microscopy of the sample under consideration reveals an averaged island base width $w$ of $130 \mathrm{~nm}$ and a corresponding averaged height $h$ of $65 \mathrm{~nm}$, see Fig. 1(a). The resulting geometrical aspect ratio $w / h$ of $\approx 2$ indicates a very effective surface minimization during island growth from a liquid phase. ${ }^{19}$ Nearly all islands arrange themselves laterally into island chains that are oriented along the crystallographic soft $\langle 100\rangle$ directions. Recently we have demonstrated that the strain energy distribution in the vicinity of a single island and an island dimer enforces the selfassembling into extended chains. ${ }^{3}$ Eventually with increasing island density a chessboard like arrangement is observed [see, e.g., the square to the lower left in Fig. 1(a)]. In case of 


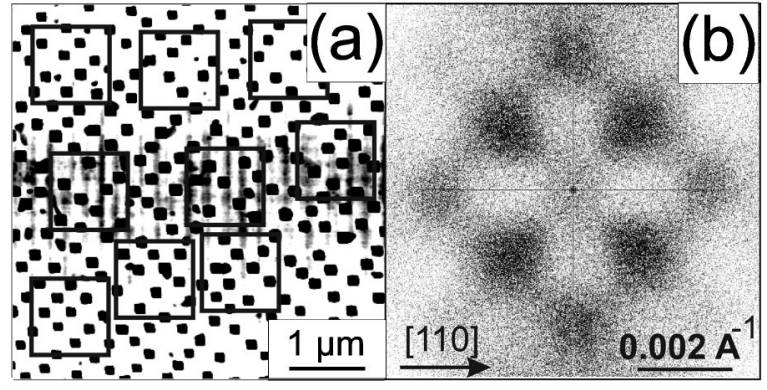

FIG. 1. (a) Atomic force micrograph of the investigated sample. The squares enclose various subensembles containing nine to ten islands. (b) Fourier transform (power spectrum) as derived from the micrograph.

low germanium content this type of arrangement can be directly attributed ${ }^{20}$ to a prepattern called rippling, which consists of shallow surface undulations along the $\langle 100\rangle$ directions. However, we could not detect any initial growth stage and only the final island morphology can be observed. In Fig. 1(b) the peaks in the Fourier transform (power spectrum) of the atomic force micrograph in Fig. 1(a) clearly reveal lateral positional correlation along both the $\langle 110\rangle$ and the $\langle 100\rangle$ directions.

\section{EXPERIMENT}

The scattering experiment has been performed at BW2 station (HASYLAB) using monochromatic $\mathrm{x}$ rays $(\lambda$ $=1.54 \AA$ ) and a linear position sensitive detector (PSD) for analyzing the direction of the scattered radiation. The wavelength resolution of $\Delta \lambda / \lambda=10^{-4}$ results in a longitudinal coherence length $\Lambda_{\text {long }}=\lambda^{2} / 2 \Delta \lambda$ of $\approx 750 \mathrm{~nm}$. Usually, the spatial coherence length of the incoming beam is determined by calculating the effective aperture seen when looking from a fixed point of the sample back to the source. Analogously the detector aperture, that is, the effective aperture seen when looking from a fixed point of the sample at the smallest resolution element of the PSD (which plays the role of an effective source size) has to be taken into account. In both cases the effective spatial coherence length is given by $\Lambda_{\text {spat }}=R \lambda / d$, where $d$ is given either by the X-ray source size or the smallest resolution element of the PSD, and $R$ is the distance between sample and source/detector. In the experimental setup used here the spatial coherence length is mainly determined by the detector spatial resolution. Using a spatial resolution of the PSD of $100 \mu \mathrm{m}$ finally results in an effective spatial coherence length of $\approx 1500 \mathrm{~nm}$, which is considerably smaller than the source-related coherence length of synchrotron radiation.

\section{RESULTS AND DISCUSSION}

Figure 2(e) depicts the measured diffusely scattered intensity in the vicinity of the 004 reciprocal lattice point within a scattering plane, which contains the [100] direction. Hence pronounced facet truncation rods originating from the island $\{111\}$ facets do not contribute to the plotted distribution. The entire cloud of diffuse intensity is related to the scattering from a particular island type. However, a remarkable impact of the positional correlation becomes obvious

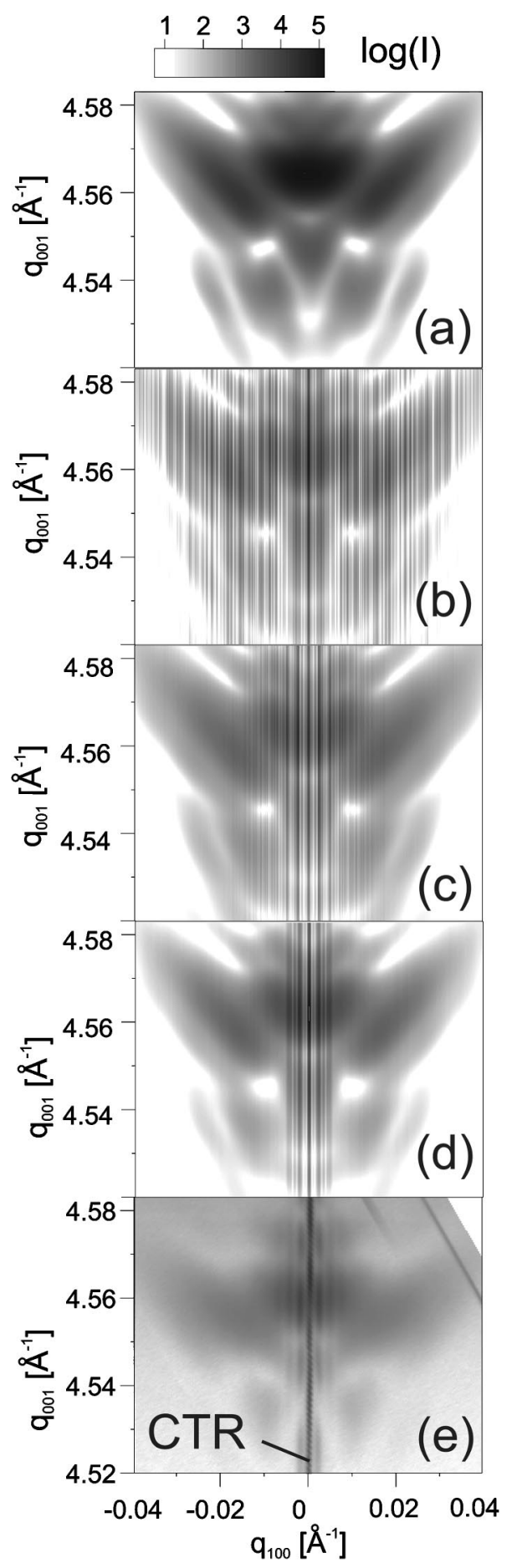

FIG. 2. Simulated diffusely scattered intensity around the symmetrical 004 reciprocal lattice point of a single island (a) and out of various island ensembles (b-d) compared with measurement (e).

through the presence of distinct correlation satellites up to third order in the nearest vicinity of the $\mathrm{Si}(001)$ crystal truncation rod (CTR). The averaged separation between them amounts to $0.0022 \AA^{-1}$. Direct scanning probe techniques as AFM suffer from comparatively less statistics, thus only the first order satellites appear in the power spectrum [Fig. 1(b)]. Corresponding peak separations amount to $\Delta q_{\langle 100\rangle}$ $=0.0020 \AA^{-1}$ and $\Delta q_{\langle 110\rangle}=0.0027 \AA^{-1}$, which roughly confirms the relationship $\sqrt{2} \times \Delta q_{\langle 100\rangle}=\Delta q_{\langle 110\rangle}$ due to the par- 
ticular cross-like pattern. These values correspond to mean lateral island-island distances of $d_{\langle 110\rangle}=233 \mathrm{~nm}$ and $d_{\langle 100\rangle}$ $=314 \mathrm{~nm}$.

Details of the underlying simulation procedure for the scattering by a single island have been described elsewhere ${ }^{11}$ and will be reported here only in brief. The numerical treatment considers the elastic strain field of the structure as calculated by the finite element method and the diffuse scattering is then simulated within the kinematical scattering approximation. We have demonstrated that a vertical germanium profile with a germanium content of $25 \%$ within the lower third of an island and $30 \%$ germanium above fits the experimentally observed features very well [see Fig. 2(a)] except for the lateral correlation peaks on both sides of the CTR, which are missing in the simulation for a single island. Here we will mainly focus on lateral positional island correlation and its impact on the diffusely scattered intensity.

For a particular island ensemble $E$ with $m$ equivalent objects the diffusely scattered intensity $I_{E}$ can be expressed as a simple product

$$
I_{E} \propto\left|A^{d i f f}(\mathbf{q}) \sum_{m \in E} e^{i \mathbf{q} \mathbf{R}_{m}}\right|^{2}=\left|A^{d i f f}(\mathbf{q})\right|^{2} G(\mathbf{q}),
$$

where $A^{\text {diff }}(\mathbf{q})$ denotes the diffusely scattered amplitude caused by a single object, $\mathbf{R}_{m}$ points to its actual position and $G(\mathbf{q})$ refers to the in-plane interference function. Thus, the calculation of the diffusely scattered intensity from an island ensemble $E$ requires adding over the index $m$ referring to the actual island positions therein. However, this coherent summation presumes perfect lateral coherence of the x-ray beam and complete information on island positions.

For the following simulations island positions are taken from AFM micrographs as depicted in Fig. 1(a). With an ensemble containing 33 islands-corresponding to a sample area of $2 \times 2 \mu \mathrm{m}^{2}$ - and coherent summation the simulation in Fig. 2(b) shows extremely pronounced speckles. Moreover, since all island positions are within a plane, the interference function does not depend on $q_{001}$. Even if the scattering of several thousand islands is considered the simulated intensity distribution (not shown here) still exhibits a complicated speckle pattern.

Obviously, the finite experimental coherence length has to be taken into account. This can be done by a partially coherent summation over areas corresponding with the experimental lateral coherence length $\Lambda$ and a subsequent incoherent (intensity) summation over many of those coherent summations:

$$
I_{E} \propto\left|A^{\operatorname{diff}}(\mathbf{q})\right|^{2} \sum_{\forall n}\left|\sum_{m \in E_{n}} e^{i \mathbf{q} \mathbf{R}_{m}}\right|^{2},
$$

$m$ subscribes the position within a coherently illuminated ensemble and $n$ indicates the different ensembles.

For the simulation in Fig. 2(c) square areas of $1 \times 1 \mu \mathrm{m}^{2}$, each enclosing nine to ten islands, have been chosen from Fig. 1(a) as indicated there. In accordance with Eq. (2) amplitudes are summed coherently within those areas and the corresponding intensities of all these areas are added. Indeed, the speckle pattern appears significantly suppressed as compared with Fig. 2(b). However, remarkable differences

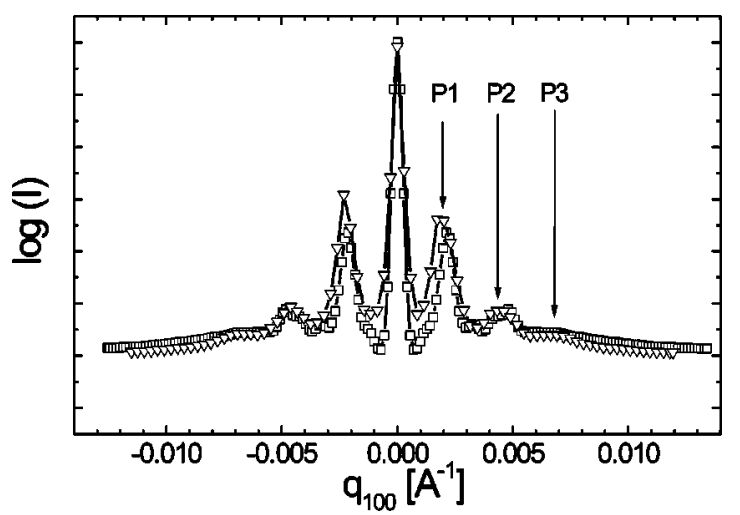

FIG. 3. Sections of the simulated (squares) and measured (triangles) intensity distribution shown in Figs. 2(d) and 2(e) at $q_{001}=4.560 \AA^{-1}$.

between measurement and simulation remain. This is related to the comparatively small number of different ensembles.

In principle, it would be possible to extract much more ensembles from a sufficiently large number of AFM micrographs. There is, however, a simpler way to improve simulations. We have numerically generated child-ensembles from those in Fig. 1(a) by adding an offset $\Omega$ along the $\langle 100\rangle$ directions to each island position randomly distributed within a range of $\pm 50 \mathrm{~nm}$. With a total number of only 1000 child ensembles the speckle pattern can be completely suppressed, Fig. 2(d). Figure 3 depicts one-dimensional intensity cuts at $q_{001}=4.560 \AA^{-1}$ through measurement and the theoretically derived distributions in Figs. 2(e) and 2(d), respectively. The numerical treatment reproduces both positions as well as corresponding intensity ratios of the satellites P1 through P3.

To implement a certain, even though small size distribution, amplitudes of two more similar island types (123.5 and $136.5 \mathrm{~nm}$ in island base) have been considered. Despite this, the resulting diffuse scattering from a single island (not shown here) does not show a significant impact of the finite size distribution. However, the interference function $G(\mathbf{q})$ becomes dependent on $q_{001}$.

\section{CONCLUSIONS}

In summary, the investigated $\mathrm{SiGe}$ nanoscale islands grown by means of liquid phase epitaxy on $\mathrm{Si}(001)$ are made of truncated pyramids with $\{111\}$ side and an (001) top facet forming exclusively a single island type. Moreover they exhibit a pronounced lateral positional correlation along the $\langle 100\rangle$ and $\langle 110\rangle$ directions. In order to probe this selforganized lateral arrangement we have applied both, direct imaging by atomic force microscopy as well as high resolution x-ray diffraction. We have demonstrated that a detailed analysis of the $\mathrm{x}$-ray diffuse scattering pattern requires careful consideration of the coherence properties of $\mathrm{x}$ rays. Although the diffraction experiment probes a sample area of about one $\mathrm{mm}^{2}$, thus an ensemble which contains $\approx 10^{7}$ equivalent islands, the scattering has to be considered coherent within much smaller areas definded by the experimental coherence length. A numerical method has been developed to implement the impact of lateral positional correlation onto the scattering pattern. This approach takes the finite experi- 
mental coherence length correctly into account by applying a partially coherent and partially incoherent superimposition of scattering amplitudes of single islands.

\section{ACKNOWLEDGMENTS}

The authors would like to thank W. Drube and $\mathrm{H}$. Schulte-Schrepping from BW2 station (HASYLAB) for the assistance during measurements. The sample preparation by $\mathrm{H}$. Wawra at the Institut für Kristallzüchtung in Berlin is gratefully acknowledged. This work was financially supported by the Deutsche Forschungsgemeinschaft under contract No. KO1510/2 and the Sonderforschungsbereich 296.

${ }^{1}$ D. Bimberg, M. Grundmann, and N. N. Ledentsov, Quantum Dot Heterostructures (Wiley, Chichester, NY, 1999).

${ }^{2}$ M. Meixner, E. Schöll, M. Schmidbauer, H. Raidt, and R. Köhler, Phys. Rev. B 64, 245307 (2001).

${ }^{3}$ M. Hanke, H. Raidt, R. Köhler, and H. Wawra, Appl. Phys. Lett. 83, 4927 (2003).

${ }^{4}$ Q. Xie, A. Madhukar, P. Chen, and N. Kobayashi, Phys. Rev. Lett. 75, 2542 (1995).

${ }^{5}$ G. Springholz, V. Holý, M. Pinczolits, and G. Bauer, Science 282, 734 (1998).

${ }^{6}$ A. Rosenauer, Transmission Electron Microscopy of Semiconductor Nanostructures: Analysis of Composition and Strain State, Springer Tracts in
Modern Physics, Vol. 182 (Springer, Berlin, 2003).

${ }^{7}$ M. Schmidbauer, T. Wiebach, H. Raidt, M. Hanke, R. Köhler, and H. Wawra, Phys. Rev. B 58, 10523 (1998).

${ }^{8}$ M. Rauscher, R. Paniago, H. Metzger, Z. Kovats, J. Domke, J. Peisl, H. D. Pfannes, J. Schulze, and I. Eisele, J. Appl. Phys. 86, 6763 (1999).

${ }^{9}$ A. Hesse, J. Stangl, V. Holý, T. Roch, G. Bauer, O. Schmidt, U. Denker, and B. Struth, Phys. Rev. B 66, 085321 (2002).

${ }^{10}$ J. Stangl, A. Hesse, V. Holý, Z. Zhong, G. Bauer, U. Denker, and O. G. Schmidt, Appl. Phys. Lett. 382, 2251 (2003).

${ }^{11}$ M. Hanke, M. Schmidbauer, D. Grigoriev, P. Schäfer, R. Köhler, A.-K. Gerlitzke, and H. Wawra, Phys. Rev. B 69, 075317 (2004).

${ }^{12}$ A. A. Darhuber, P. Schittenhelm, V. Holý, J. Stangl, G. Bauer, and G. Abstreiter, Phys. Rev. B 55, 15652 (1997).

${ }^{13}$ I. Kegel, T. H. Metzger, J. Peisl, P. Schittenhelm, and G. Abstreiter, Appl. Phys. Lett. 74, 2978 (1999).

${ }^{14}$ K. Zhang, C. Heyn, W. Hansen, T. Schmidt, and J. Falta, Appl. Phys. Lett. 76, 2229 (2000)

${ }^{15}$ V. Chamard, T. H. Metzger, E. Bellet-Amalric, B. Daudin, C. Adelmann, H. Mariette, and G. Mula, Appl. Phys. Lett. 79, 1971 (2001).

${ }^{16}$ V. Holý, T. Roch, J. Stangl, A. Daniel, G. Bauer, T. H. Metzger, Y. H. Zhu, K. Brunner, and G. Abstreiter, Phys. Rev. B 63, 205318 (2001).

${ }^{17}$ M. Hanke, M. Schmidbauer, R. Köhler, H. Kirmse, and M. Pristovsek, J. Appl. Phys. 95, 1736 (2004).

${ }^{18}$ R. T. Lechner, T. U. Schülli, V. Holý, G. Springholz, J. Stangl, A. Raab, G. Bauer, and T. H. Metzger, Appl. Phys. Lett. 84, 885 (2004).

${ }^{19}$ M. Hanke, M. Schmidbauer, R. Köhler, F. Syrowatka, A.-K. Gerlitzke, and T. Boeck, Appl. Phys. Lett. 84, 5228 (2004).

${ }^{20}$ W. Dorsch, B. Steiner, M. Albrecht, H. P. Strunk, H. Wawra, and G. Wagner, J. Cryst. Growth 183, 305 (1998). 